\title{
Toward Realistic Large-Area Cell Membrane Mimics: Excluding Oil, Controlling Composition, and Including lon Channels
}

Journal Article

Author(s):

Beltramo, Peter J.; Scheidegger, Laura; Vermant, Jan

Publication date:

2018-05-22

Permanent link:

https://doi.org/10.3929/ethz-b-000268448

Rights / license:

In Copyright - Non-Commercial Use Permitted

Originally published in:

Langmuir 34(20), https://doi.org/10.1021/acs.langmuir.8b00837 


\title{
Towards realistic large area cell membrane
}

\section{mimics: Excluding oil, controlling composition and including ion channels}

\author{
Peter J. Beltramo, ${ }^{* \dagger}$, Laura Scheidegger, ${ }^{\ddagger}$ and Jan Vermant ${ }^{\ddagger}$ \\ $\dagger$ Department of Chemical Engineering, University of Massachusetts Amherst, Amherst, MA \\ 01003, USA. \\ $\ddagger$ Department of Materials, ETH Zürich, Vladimir-Prelog-Weg 5, 8093 Zürich, Switzerland. \\ E-mail: pbeltramo@umass.edu
}

\begin{abstract}
Capacitance measurements provide unique insights into the thickness, compressibility, and composition of large area membrane bilayers and are used here in addition to demonstrate the successful incorporation of model ion channels. The simultaneous ability to control the bilayer size and manipulate tension, optically monitor and electrically stimulate free-standing membranes enables precise determination of their specific capacitance and thickness across a wide range of areas. We confirm that membranes formed by this recently developed technique have capacitive properties similar to those formed by existing protocols, including solvent-free approaches, and discuss the effect using either hexadecane or squalene as the oil solvent. The results obtained here are relevant for other methods where lipid membranes are reconstituted from a bulk oil solvent. Since biological membranes have a diverse phospholipid profile, we show that the technique can successfully reconstitute membranes with binary composition mixtures. As an outlook, we show the capability for model membrane proteins, specifically
\end{abstract}


$\alpha$-hemolysin and alamethicin, to be incorporated into the formed bilayers and measure ion transport.

\section{Introduction}

The development of model cellular membranes is crucial for fundamental investigations of biological phenomena, ranging from membrane protein pore formation and biomolecule transport ${ }^{1,2}$ to bilayer elasticity ${ }^{3}$ and lipid raft formation. ${ }^{4}$ Such efforts can then be exploited to engineer new advances in biosensing, targeted drug delivery and therapeutics, and biomimetic technologies. The model membranes used must replicate key aspects of the actual cell or organelle membranes with high fidelity in order to translate results from in vitro to in vivo systems.

Significant progress has been made to create model biomembranes, however many of the existing methods have limitations. Techniques relying on the formation of black lipid membranes $(B L M s)^{5-7}$ over apertures can only examine whatever state is produced by the spontaneous bilayer self-assembly, and the resulting films are often unstable. Droplet-interface bilayers (DIBs) ${ }^{8-10}$ are excellent for making droplet networks and detecting small transmembrane fluxes, but not conveniently imaged in the plane of the bilayer, unless in some cases a hydrogel support is used, ${ }^{11,12}$ and require micromanipulation equipment. ${ }^{13} \mathrm{~A}$ common critique of model bilayer studies that rely on forming a membrane from an oil solvent, such as the BLM, DIB, and the present method, is the presence of residual oil in the bilayer. ${ }^{12-15}$ Strategies to minimize residual oil in the bilayer include the solvent freeze-out method, ${ }^{16}$ fluorosiliconizing BLM apertures, ${ }^{17}$ and increasing the chain length of the oil solvent, ${ }^{12}$ in particular by using squalene. ${ }^{18}$ We will quantify the effectiveness of the latter approach in the

present work by measuring the membrane specific capacitance. Supported bilayers ${ }^{19-22}$ are easily imaged and very amenable to scattering techniques, yet the influence of the support is ever-present and it is difficult to incorporate proteins, although there are some recent suc- 
cesses. ${ }^{23,24}$ Giant unilamellar lipid vesicles ${ }^{25-30}$ are solvent-free, but there are limitations to the aqueous phase chemistry and the interior compartment is not easily changed. A valuable advantage of GUVs are the ability to perform pipette aspiration to change the membrane tension from $0.001 \mathrm{mN} / \mathrm{m}$ to $10 \mathrm{mN} / \mathrm{m}$ to determine the elastic moduli, bending modulus, and area compressibility modulus. ${ }^{31}$

The majority of studies involving model membranes only focus on symmetric membranes dividing a uniform aqueous solution, however one key characteristic of cell membranes is the presence of a transmembrane electric potential. This potential is approximately 100 $\mathrm{mV}$ in magnitude and arises due to a variety of factors operating in tandem, namely the action of ion pumps to maintain an osmotic pressure difference, the presence of charged membrane proteins, membrane curvature, and asymmetry of charged lipid composition. ${ }^{32}$ Therefore, it is important to examine asymmetric membranes, ${ }^{33-35}$ those with an osmotic pressure difference, ${ }^{22,36,37}$ or those with an external transmembrane potential, in order to faithfully determine membrane behavior under physiologically relevant conditions.

We have recently developed a technique to create large-area model biomembranes (LAMBs) ${ }^{38,39}$ by adopting a microfluidic thin film balance technique ${ }^{40}$ and improving the pressure control and chip functionlization to stabilize phospholipid membranes in aqueous solution. Our previous work demonstrated the utility of the LAMB platform in optically imaging phase separating lipid bilayers with high resolution due to the planar nature of the membranes and the unique ability to control membrane tension from $0.5-5 \mathrm{mN} / \mathrm{m}$ on a planar, free-standing bilayer. This paper is focused on demonstrating how more realistic cell membrane mimics with controlled composition, tension and thickness can be made by determining the membrane specific capacitance and thickness, and proof-of-principle ion channel recordings. Since the LAMB technique relies on a lipid bilayer formed from a bulk oil solution, particular attention is given to the effect of oil, both on the as formed bilayer thickness and also the dynamic compression of the membrane due to an external potential. Changing the molecular weight of the oil to change the length of the solvent molecules relative 
to the thickness of the bilayer is found to be key to oil inclusions. Hexadecane $\left(\mathrm{C}_{16} \mathrm{H}_{34}, \mathrm{M}_{w}=\right.$ $226 \mathrm{~g} / \mathrm{mol})$ swells the bilayer, whereas squalene $\left(\mathrm{C}_{30} \mathrm{H}_{50}, \mathrm{M}_{w}=411 \mathrm{~g} / \mathrm{mol}\right)$ gets excluded. In addition, we demonstrate the ability to control bilayer composition through adsorption from the bulk oil using a series of binary lipid mixtures. The paper is organized as follows: first, we introduce the background regarding capacitance measurements on lipid bilayers. Then, the successful formation of bilayers with specific capacitance approaching that of solvent free membranes is demonstrated using squalene as the oil phase before the response of the membrane to external fields as a function of oil solvent is presented. It is shown that using squalene as the oil effectively results in bilayers with specific capacitance approaching the solvent-free values. The thickness dependence of binary mixtures is then used to argue that what is in the final membrane corresponds to the lipid concentrations in the bulk oil, with a comparison to scattering techniques and a critical discussion of the hydrocarbon dielectric constant. Finally, the successful incorporation of $\alpha$-hemolysin and alamethicin ion channels is shown.

\section{Background}

The phospholipid bilayer consists of a hydrophobic core hydrocarbon layer of low dielectric constant $\left(\varepsilon_{r}=2-3\right)$ sandwiched around an interfacial phospholipid headgroup region of higher dielectric constant $\left(\varepsilon_{r}=10-30\right) .{ }^{41}$ Altogether, the membrane separates an aqueous solution of much higher dielectric constant $\left(\varepsilon_{\mathrm{H}_{2} \mathrm{O}} \approx 80\right)$. The bilayer can therefore be modelled as a capacitor and the capacitance of the membrane can be written as

$$
C=\varepsilon_{r} \varepsilon_{0} \frac{A}{d}
$$

where $\varepsilon_{r}$ is the dielectric constant of the hydrophobic core, $\varepsilon_{0}$ is the permittivity of free space, $A$ is the area of the bilayer, and $d$ is the thickness of the hydrophobic core. It is very difficult to isolate and measure the dielectric constant of the phospholipid headgroup region, ${ }^{41}$ but 
its contribution to the overall capacitance of the membrane is negligible ${ }^{42,43}$ and therefore neglected when calculating the thickness of membranes. ${ }^{12}$ This is also commonly the case in thin film interferometric studies of surfactant films in air, where the film thickness calculated using a uniform refractive index is reported. ${ }^{39,44-46}$

Often it is effective to report data in terms of the specific capacitance, which is defined as the ratio of capacitance to area,

$$
C_{m}=\frac{C}{A}=\frac{\varepsilon_{r} \varepsilon_{0}}{d}
$$

The advantage of reporting data in terms of the specific capacitance is that it comes completely from measured quantities: the capacitance from an electrical method and the area determined optically. One of the advantages of the LAMB technique is the ease with which the area can be quantified. The specific capacitance can be used to compare the relative thickness of two membranes using the following equation,

$$
\frac{C_{m, 1}}{C_{m, 2}}=\frac{d_{2}}{d_{1}},
$$

where the subscripts 1 and 2 refer to two membranes with the same dielectric constant.

To convert capacitance data to a thickness,

$$
d=\varepsilon_{r} \varepsilon_{0} \frac{A}{C}
$$

one must consider the actual dielectric constant of the membrane. Dielectric constants ranging from $2.1^{47}$ to $2.8^{48}$ have been used previously. In the results presented below, we have chosen a dielectric constant of $\varepsilon_{r}=2.5$ because it successfully brings our specific capacitance data for DOPC bilayers formed from squalene in concert with independent determination of the hydrophobic bilayer thickness by small-angle X-ray scattering (SAXS). ${ }^{49-53}$ Back calculating the specific capacitance using Eqn. 2 with a lower dielectric constant of $\varepsilon_{r}=2.2$ 
and the SAXS bilayer thickness results in a lower specific capacitance than expected for solvent-free bilayers.

Similarly to BLMs, membranes formed using the LAMB technique possess an annulus of bulk oil surrounding the circular bilayer. However, it has been previously shown that the annulus contributes a negligible capacitance to the overall bilayer capacitance in this size regime. ${ }^{54}$ As discussed below, this contribution is roughly $10 \mathrm{pF}$, which is $0.2-3 \%$ of the overall measured capacitance in our experiments.

Applying an external potential allows for the determination of the Young's (elasticity) modulus perpendicular to the membrane surface. This is known as the electrostriction method since the applied potential will cause charge separation between either side of the membrane resulting in an additional dipole and mechanical force that will cause the membrane to compress (become thinner) as a result. It has recently been shown that this potential can cause phase separation in model membranes, ${ }^{55}$ but in this work we focus on mixtures well within the miscibility region. According to Hianik et al. ${ }^{56},{ }^{57}$, the Young's modulus can be written as

$$
E_{\perp}=-p /(\Delta d / d)
$$

where $p$ is the pressure induced by the external potential and $\Delta d$ is the change in membrane thickness. The pressure on the membrane is a function of the applied voltage, $V$, and the membrane specific capacitance as

$$
p=\frac{C_{m} V^{2}}{2 d}
$$

Rearranging, the membrane thickness decreases with the square of the applied electric field,

$$
\Delta d=\frac{C_{m} V^{2}}{2 E_{\perp}}
$$

From Equation 7 it is obvious that by measuring the thickness decrease, $\Delta d$, as a function of applied voltage, $V$, one can readily determine the modulus. 


\section{Experimental}

\section{Materials}

The phospholipids 1,2-dioleoyl-sn-glycero-3-phosphocholine (DOPC), 1,2-dioleoyl-sn-glycero3-phosphoglycerol (DOPG), 1,2-diphytanoyl-sn-glycero-3-phosphocholine (DPhPC), and 1,2dilauroyl-sn-glycero-3-phosphocholine (DLPC) were obtained from Avanti Polar Lipids. Squalene, n-hexadecane, and octadecyltrichlorosilane (OTS) were purchased from Acros Organics. $\mathrm{NaCl}$ (99.99\%, metals basis) and $\mathrm{NaHCO}_{3}$ were obtained from Alfa Aesar. $\mathrm{CaCl}_{2}$, $\alpha$-hemolysin, and alamethicin was purchased from Sigma-Aldrich. Ultra-pure water (MilliQ, Merck-Millipore, resistivity $<18.2 \mathrm{M} \Omega \cdot \mathrm{cm})$ was used to make all buffers.

\section{Sample preparation}

Phospholipids stored in chloroform are dried under nitrogen, placed under vacuum, and resuspended in either squalene or n-hexadecane following the same procedure outlined previously. ${ }^{38}$ To make binary mixtures, monocomponent lipid samples are prepared at a concentration of $4 \mathrm{mM}$ in oil and subsequently combined to the desired ratio. An aqueous salt buffer of $150 \mathrm{mM} \mathrm{NaCl}, 2 \mathrm{mM} \mathrm{CaCl}_{2}, 0.2 \mathrm{mM} \mathrm{NaHCO}_{3}$ was prepared and filtered with a $0.2 \mu \mathrm{m}$ pore filter before use. Solutions of $\alpha$-hemolysin were prepared in the salt buffer at a concentration of $4.55 \mu \mathrm{M}$ and diluted upon addition to an already formed bilayer. Alamethicin was dissolved in ethanol at a concentration of $10 \mathrm{nM}$ before being added to an already formed bilayer.

\section{Experimental setup for capacitance measurements}

The recently developed thin film balance technique to create large-area model biomembranes $(\mathrm{LAMBs})^{38}$ is extended here to accomodate simultaneous optical and electrical recording (Fig 1, top). We therefore highlight the changes necessary to perform capacitance measurements since preparation of the microfluidic chip and the pressure control system are as described 
previously. A patch-clamp amplifier (EPC10, Heka) with an S-Probe headstage are used for current and voltage measurements. A custom sample chamber is made such that the cis and trans sides of the bilayer can be electrically isolated with an equal hydrostatic pressure imposed on the bilayer. To accomplish this, a small hole is drilled connecting the bottom reservoir to a larger chamber open to air. High viscosity vacuum grease is used to ensure the microfluidic chip is in contact with the teflon sample chamber and there are no material or electrical connections between the two chambers. Patchmaster software (Heka) is used to control the recordings. Capacitance measurements are calibrated using a model circuit (MC-10, Heka) and performed using the Lock-In configuration with an applied AC voltage of $1 \mathrm{kHz}$ with a $\pm 10 \mathrm{mV}$ amplitude centered at a desired offset voltage. The current response is filtered by two onboard Bessel filters in the EPC 10 of $10 \mathrm{kHz}$ and $2.9 \mathrm{kHz}$, respectively.
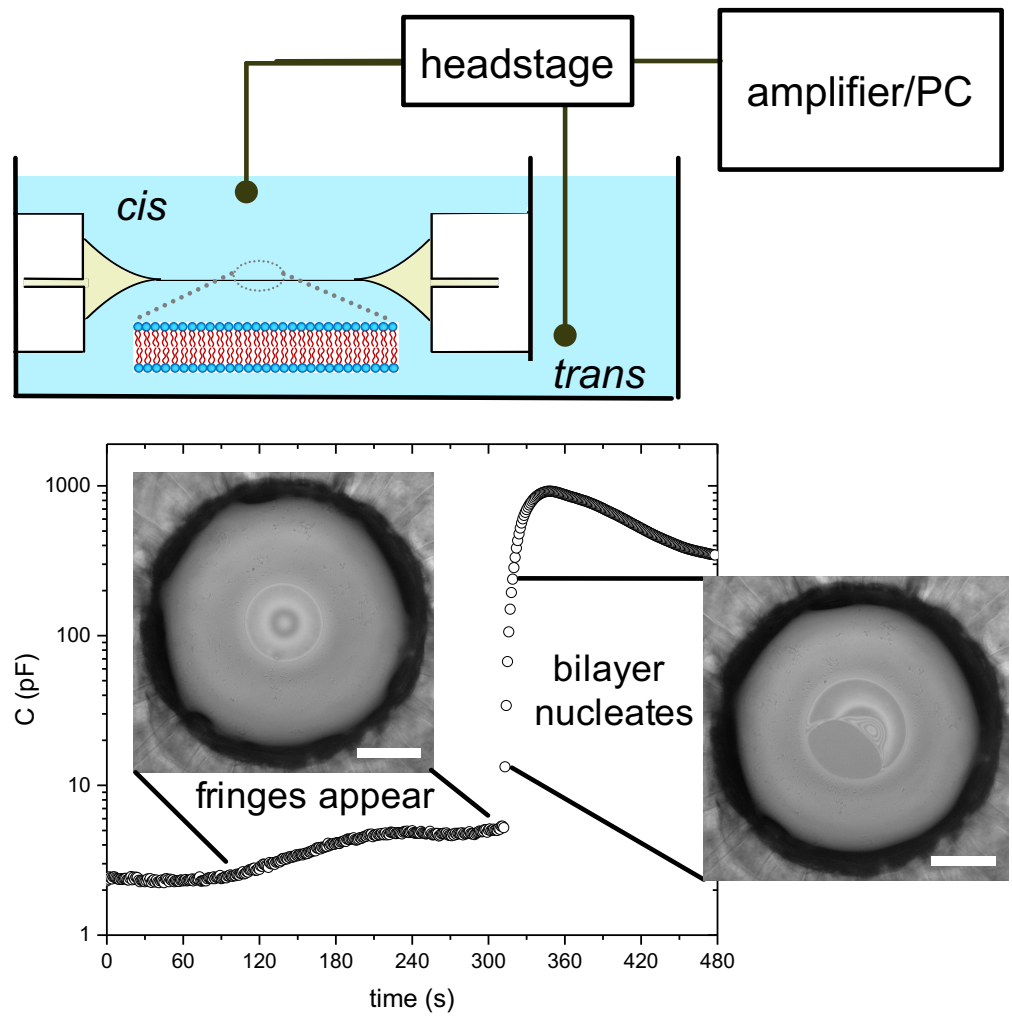

Figure 1: Top: Schematic of the experimental setup to perform capacitance measurements with the LAMB technique. Bottom: Capacitance of $2.5 \mathrm{mg} / \mathrm{mL}$ DOPC in squalene as the film thins and ultimately forms a bilayer, with representative images of the film as interferometric fringes appear and the bilayer nucleates (scale bar $=0.2 \mathrm{~mm}$ ). 
For ion channel recordings, custom chart recording functions are used. The sample chamber is placed in a Faraday cage with openings for tubing and the microscope objective, which currently brings the noise level of the system to approximately $15 \mathrm{pA}$. Simultaneously to the electrical recordings, the bilayer is imaged on an upright microscope (Nikon Eclipse FN1) in reflection using a monochromatic LED light source (Spectra-X) with a 10x long working distance objective and a Hamamatsu ORCA-Flash4.0 CMOS camera.

\section{Results and Discussion}

\section{Static effects of oil}

We first focus on the results of monocomponent DOPC bilayers formed from two oils, nhexadecane and squalene. As Figure 1 shows, the capacitance is very low $(\sim 2 \mathrm{pA})$ while a thick oil film is present in the microfluidic orifice. When interferometric fringes appear due to the thinning of the film, the capacitance steadily increases to a value of $7-12 \mathrm{pF}$, depending on the extent of the fringes. Finally, when the bilayer first forms the capacitance rapidly increases orders of magnitude due to the nanometer scale thickness of the bilayer. The capacitance during thinning is a proxy for the contribution of the annulus to the overall capacitance. However, the $7-12 \mathrm{pF}$ measured during thinning should be an upper bound, since both the Plateau border and the thin area where interference fringes appear contribute to the capacitance.

By monitoring the capacitance while simultaneously imaging the size of the bilayer, the specific capacitance (Eqn 2) can easily be calculated, and likewise the film thickness (Eqn 4) after assuming a value for the bilayer dielectric constant. Figure 2A shows the capacitance, specific capacitance, and thickness for a DOPC bilayer formed with squalene used as the oil. It is apparent that the specific capacitance and thickness do not change appreciably during expansion/compression of the bilayer. Our previous work observed an increase in membrane tension as the bilayer area expanded (from $0.5-4 \mathrm{mN} / \mathrm{m}$ ), however a tension change of 

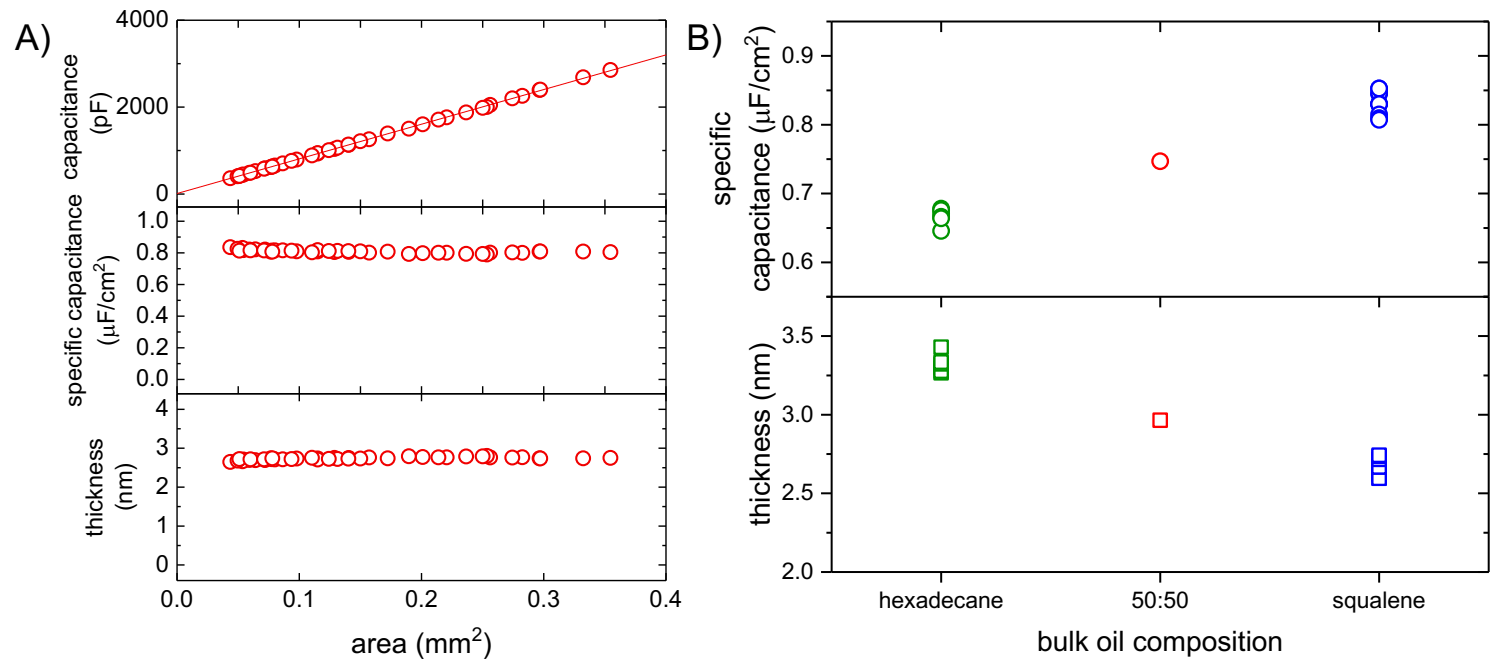

Figure 2: A) Capacitance, specific capacitance, and bilayer thickness as a function of bilayer area for $5 \mathrm{mg} / \mathrm{mL}$ DOPC in squalene. B) Specific capacitance and bilayer thickness for independent bilayers formed from varying oil solvents. The specific capacitance and thickness are independent of DOPC concentration in the bulk oil.

this magnitude does not have a notable effect on the bilayer thickness. The independence of specific capacitance (within measurement accuracy) with changing film area was observed across all experiments, including with multiple bilayers formed from independent samples, different lipid concentrations in oil, and different oils.

The average specific capacitance and the calculated thickness for DOPC bilayers formed from three different oil conditions is shown in Figure 2B. Each data point shown in Figure $2 \mathrm{~B}$ is the average value for an independent lipid bilayer formation, with 6 bilayers formed from hexadecane and 9 bilayers formed from squalene. Using hexadecane, independent bilayers form repeatable specific capacitances of $0.67 \pm 0.01 \mu \mathrm{F} / \mathrm{cm}^{2}$ from which a (hydrophobic) thickness of $3.32 \pm 0.06 \mathrm{~nm}$ is obtained, using an epsilon value of $\varepsilon_{r}=2.5$ for the dielectric constant of the hydorophobic core of the DOPC bilayer. The shorter chain hexadecane molecules remain in the bilayer and cause the measured thickness to increase, similar to what was found by Gross et al. ${ }^{12}$. When squalene, a larger molecular weight oil, is used, the bilayer thickness is $25 \%$ thinner, $2.66 \pm 0.05 \mathrm{~nm}$, as obtained from the increase in specific capacitance to $0.83 \pm 0.02 \mu \mathrm{F} / \mathrm{cm}^{2}$. For comparison, SAXS experiments on solvent-free bilay- 
ers consistently report hydrophobic bilayer thickness of $2.7 \pm 0.04 \mathrm{~nm} .{ }^{49-52}$ Garten et al. ${ }^{58}$ recently performed capacitance measurements on GUVs, which are of course solvent-free bilayers. For DOPC, the measured specific capacitances using this approach on independent GUVs ranged from $0.83-0.91 \mu \mathrm{F} / \mathrm{cm}^{2}$. Therefore, despite forming bilayers from an oil solvent, the use of long-chain oils such as squalene is an effective strategy to form membranes with thicknesses and specific capacitances on par with solvent-free approaches.

\section{Dynamic effects of oil: electrostriction}

While only the static effects of oil on bilayer thickness have been examined using capacitance measurements previously, we aimed to measure the elastic properties by applying a DC electric field, which applies an electrostriction force, and monitoring the concurrent change in membrane area and capacitance. This was done by increasing the DC-voltage offset repeatedly in a step-wise manner while still measuring the capacitance using a superimposed AC signal of $1 \mathrm{kHz}$ with a $\pm 10 \mathrm{mV}$ amplitude. The DC field causes an accumulation of ions of either side of the membrane which polarize and compress the membrane. The magnitude of the electric potential across the membrane may therefore be different than that applied by the electrodes the due to ionic polarization. ${ }^{59}$ In performing these measurements, we must consider the timescales over which the membrane responds to a step change in voltage, which is related to the time scale over which ions polarize on either side of the membrane before the membrane senses the external potential, and report the thickness decrease of the membrane after it has reached a constant value. We found that it takes $5-10$ seconds for the bilayer to adjust to the increased field strength conditions, but only $1-2$ seconds to relax back when the field is turned off. An example experiment for a DOPC bilayer formed from squalene is shown in Fig 3a, where the voltage was toggled on for 20 seconds and off for 10 seconds. As the field strength increases, the area of the bilayer increases, as does the capacitance. In converting this data to the thickness, it is obvious that the majority of the capacitance increase is due to the area increase of the membrane. However, the bilayer does 

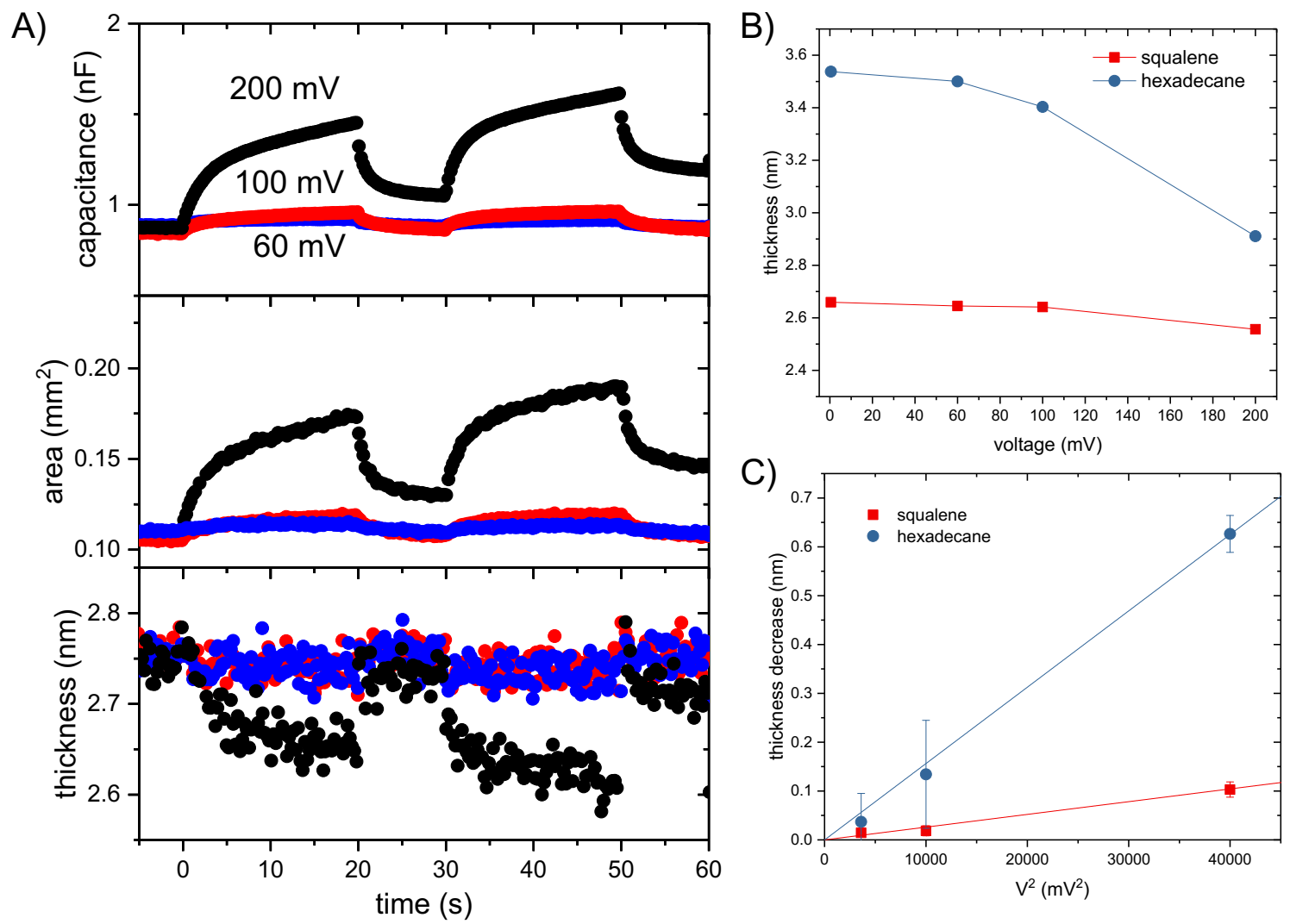

Figure 3: A) Time evolution of the bilayer response as an electric potential of varying magnitudes is repeatedly applied to a DOPC bilayer. B) The bilayer thickness decreases with increasing voltage in a manner dependent on the bulk oil composition. C) The magnitude of thickness decrease is proportional to $V^{2}$, which is used to calculate the elasticity modulus. The steeper the dependence the lower the modulus.

show a measurable thickness decrease which increases in magnitude as the field strength increases. The dynamics of these transitions are most easily seen in the data for the 200 $\mathrm{mV}$ experiment, where the bilayer thickness decreases by $\sim 0.1 \mathrm{~nm}$ after applying the voltage for 10 seconds, but returns back to its original value almost instantaneously after removing the external field. The effect of electrolyte on the temporal response to the bilayer was not explored, however it is reasonable to surmise that the polarization of ions will be a function of concentration, leading to different kinetics or compression of the bilayer.

The response of a DOPC membrane formed from either squalene or hexadecane to at least three compression cycles from an external field is summarized in Fig 3b. While the 
hexadecane forms thicker membranes, it also compresses more on a total magnitude and percentage basis, at a given voltage. This could be due to residual solvent molecules trapped in the membrane that are squeezed out upon application of the field. As mentioned in our previous work, bilayers formed by the LAMB technique can be stable up to several hours. ${ }^{38}$ The electric field does destabilize the bilayers; short pulses of voltage from $200-500 \mathrm{mV}$ often break the membrane. We have not explored the breakdown voltage-composition parameter space in detail, but doing so may yield additional information about the electromechanical properties of lipid bilayers.

The comparison between the thickness decrease, $\Delta d$ and voltage is shown in Fig. 3c. The expected $V^{2}$ dependence based on Eqn 7 is observed in both cases. The Young's modulus is therefore calculated from a linear fit to this data. The modulus calculated in this fashion is $2.16 \times 10^{5} \mathrm{~Pa}$ for DOPC bilayers in hexadecane, and increases an order of magnitude to $1.58 \times 10^{6} \mathrm{~Pa}$ for DOPC bilayers in squalene. This is notable since not only does the oil affect the magnitude of the thickness/dielectric constant, but also the compressibility response of the bilayers to external fields.

There is not a direct comparison in the literature to the experiments performed here on free-standing DOPC membranes with varying oil solvent. The closest comparison is the studies of Hianik et al. ${ }^{56}$, Hianik ${ }^{60}$, who examined the effect of oil on the elasticity modulus of black lipid membranes formed using soybean phosphatidylcholine formed on solid supports or painted on small apertures. In general, the modulus values were $\sim 10^{6}-10^{8} \mathrm{~Pa}$. The modulus values were surprisingly independent of oil for supported bilayers, but did increase with molecular weight for free-standing membranes (from $1.3 \times 10^{7}$ to $6.2 \times 10^{7} \mathrm{~Pa}$ when the oil is n-decane and n-hexadecane, respectively). Soybean phosphatidylcholine contains a mixture of fatty acids, with a major component 18:2 PC (63\%, two double bonds on each 18 carbon tail) and a significant portion (15\%) smaller chain saturated phospholipids, so although a direct comparison is not possible it is reassuring that the elasticity values reported here are the same order of magnitude. The Young's modulus for bulk linear low density 
polyethylene/wax blends is on the order of $100 \mathrm{MPa}\left(10^{8} \mathrm{~Pa}\right)^{61}$ which serves as an upper bound for the possible values and indicates the results of Hianik et al. ${ }^{56}$ may be towards the higher limit.

A major difference between our study and that of Hianik et al. ${ }^{56}$ is in the dynamics of capacitance change. Where Hianik et al. ${ }^{56}$ observed capacitance changes over the course of minutes after applying an electric field, we see the thickness plateau after approximately 10 seconds. This highlights the benefit of optically monitoring the membrane area while measuring the capacitance, since we are able to verify the bilayer thickness being constant despite the overall capacitance (membrane area) still increasing. While an exhaustive determination of the elasticity modulus of different phospholipid/oil combinations is out of the scope of this work, the take home message is clear: to determine membrane biophysical properties one must be cognizant of the effect residual oil in the membrane may have on both the static and dynamic bilayer behavior.

\section{Multicomponent lipid mixtures}

From the preceding results, it is clear that using squalene as the oil solvent results in a membrane with less residual oil, a hydrophobic thickness on par with solvent free methods, and an Young's modulus in line with previous literature results. Therefore, using squalene as the oil solvent, the LAMB technique can be extended to solvent free multicomponent lipid mixtures. Since the lipid bilayers are formed from a bulk oil solution, it is important to confirm that the final lipid composition in the membrane corresponds with the lipid composition mixed in the bulk solution.

A series of binary lipid mixtures were studied, including DOPC and either DLPC, DOPG or DPhPC. These mixtures were chosen because they either modify the hydrocarbon thickness with constant headgroup (DOPC/DLPC) or modify the phospholipid headgroup with constant hydrocarbon thickness (DOPC/DOPG). Additionally, DOPC/DPhPC was chosen due to the abundance of literature studies using DPhPC in DIBs ${ }^{12,13}$ and BLMs. ${ }^{15}$ All three 
mixtures are expected to be a single phase at room temperature.

Figure 4A shows the binary DOPC/DLPC mixture specific capacitance and thickness, with the given mole percentages calculated by the concentration of lipids in the bulk oil. The specific capacitance (thickness) increases (decreases) linearly with increasing amounts of DLPC in the membrane, indicating mixtures with the expected phospholipid bilayer composition are indeed being formed. As expected, the shorter chain DLPC contributes to a thinner lipid bilayer forming. Bilayers of pure DLPC were not able to be successfully formed, but extrapolating the data to monocomponent DLPC results in a thickness of $1.75 \mathrm{~nm}$. This is $33 \%$ shorter than DOPC, which aligns with the ratio of carbons making up the hydrophobic tails (12 for DLPC, 18 for DOPC). However, SAXS data on DLPC report a slightly thicker hydrophobic bilayer thickness of $1.95-2.09 \mathrm{~nm} .{ }^{49,50,62}$

Although the chemical nature of the fatty acid tails is quite similar between DOPC and DLPC, one could argue that there may be slight differences in dielectric constant (note this would not affect the specific capacitance). As a result, the DOPC/DOPG mixture assures a uniform dielectric constant while introducing variation in headgroup composition. Being anionic, mixtures with increasing concentration of $\mathrm{PG}$ headgroups are expected to be comparatively thicker due to increased electrostatic repulsion across the membrane. Once again, monocomponent DOPG membranes were not stable, however mixtures with up to 50 \% DOPG indicated the expected linear increase in membrane thickness (Fig. 4B). Extrapolating the linear dependence of the binary mixture thickness to $100 \%$ DOPG results in an expected membrane thickness of $2.83 \mathrm{~nm}$, in good agreement with the hydrophobic bilayer thickness of $2.77 \mathrm{~nm}$ as determined by SAXS. ${ }^{63,64}$

The final binary mixture examined was DOPC/DPhPC (Fig. 4C), which also exhibits the linear trend. There are deviating results in the literature regarding the thickness of DPhPC bilayers in comparison to DOPC bilayers measured by SAXS. Kučerka et al. ${ }^{53}$ found thicknesses of $2.75 \mathrm{~nm}$ for DPhPC and $2.68 \mathrm{~nm}$ for DOPC, ${ }^{51}$ however Valincius et al. ${ }^{48}$ reported thicknesses of $2.86 \mathrm{~nm}$ for $\mathrm{DPhPC}$ and $3.1 \mathrm{~nm}$ for DOPC, a quantitative and 

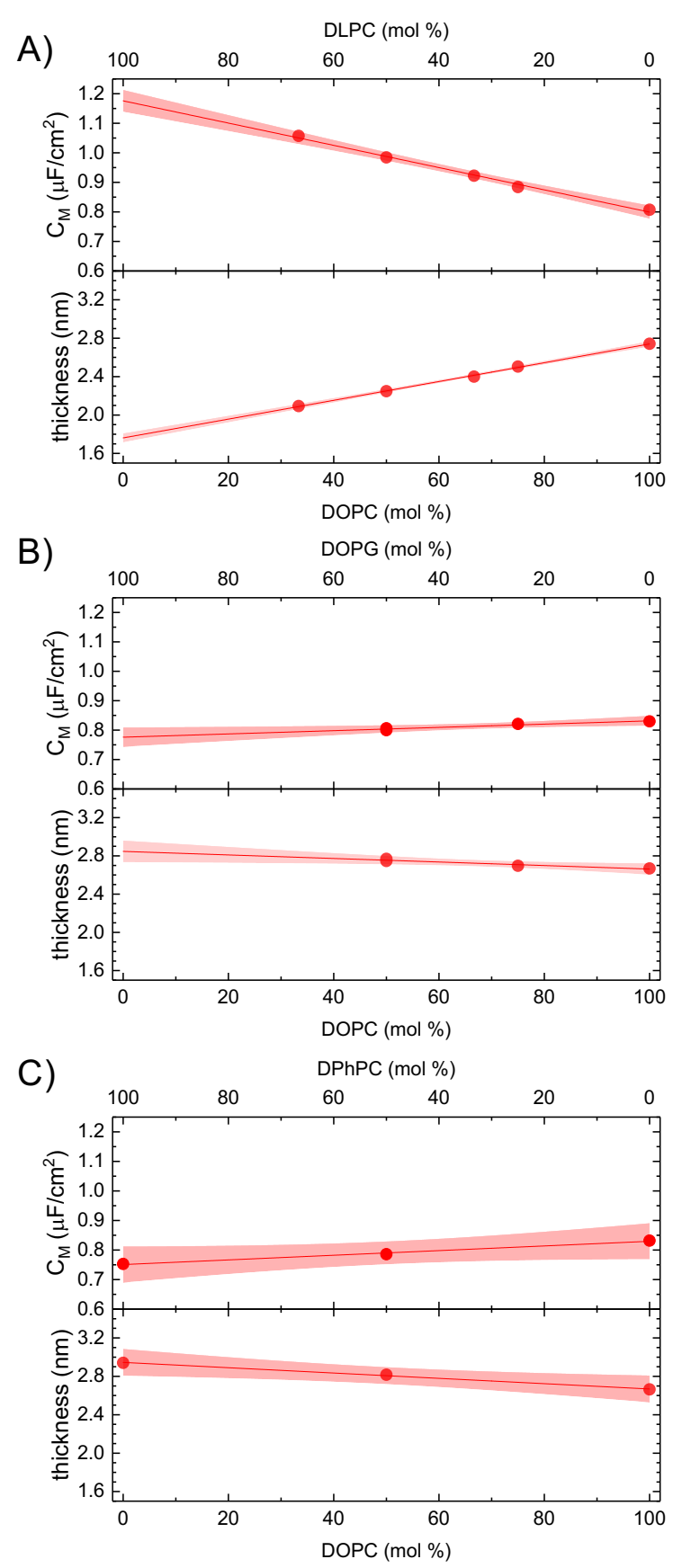

Figure 4: Binary mixtures of DOPC and A) DLPC, B) DOPG and C) DPhPC show a linear dependence with bulk lipid composition, indicating that the composition of the membrane can be described by the bulk mole fractions. Lines are linear fits to the data with $95 \%$ confidence intervals.

qualitative difference. Valincius et al. ${ }^{48}$ also measured the dielectric constant $\varepsilon_{r}=2.2$ for $\mathrm{DPhPC}$, which gives a specific capacitance of $0.68 \mu \mathrm{F} / \mathrm{cm}^{2}$. Because this value is well below 
that expected for solvent-free DPhPC bilayers, ${ }^{58}$ we have chosen to compare our data to the results of Kučerka et al. ${ }^{53}$. Our membrane thickness is slightly higher (2.95 nm vs $\left.2.75 \mathrm{~nm}\right)$ than determined by SAXS, ${ }^{53}$ and likewise the specific capacitance is lower $\left(0.75 \mu \mathrm{F} / \mathrm{cm}^{2}\right.$ vs $0.87 \mu \mathrm{F} / \mathrm{cm}^{2}$ ) than determined using GUVs. ${ }^{58}$ Lastly, by comparing the specific capacitance values one can estimate the degree to which the bilayers formed by the LAMB approach are thinner/thicker than their GUV counterparts. Assuming an equal dielectric constant, Eqn 3 can be applied, which yields a 5\% thicker bilayer for DOPC in squalene and a $16 \%$ thicker bilayer for DPhPC in squalene for the LAMB technique compared to GUVs. ${ }^{58}$ Despite having an overall shorter hydrocarbon tail (16 vs 18), perhaps the higher overall molecular weight of the chains due to the methyl groups cause DPhPC to form bilayers which have increased area per lipid molecule $\left(79 \AA^{2}\right.$ for $\mathrm{DPhPC}^{53}$ compared to $72 \AA^{2}$ for DOPC ${ }^{51}$ ) which more readily accommodate squalene.

\section{Incorporation of ion channels}

A common motivation for developing model membrane techniques is to analyze the dynamics of membrane proteins, and in doing so develop fundamental insights into their activity and leverage their behaviour for biosensing, among other applications. Here, we demonstrate the successful incorporation of two membrane proteins, $\alpha$-hemolysin and alamethicin, into DOPC membranes formed with either hexadecane or squalene. While our prior results ${ }^{38}$ and the capacitance measurements discussed earlier both indicate a true phospholipid bilayer is formed using the LAMB technique, the ability to incorporate ion channels serves as an additional confirmation and important capability for applying the platform to more complicated problems in biological physics.

Specifically, $\alpha$-hemolysin has commonly been used to validate bilayer formation since its $2.8 \mathrm{~nm} \beta$ barrel $^{65}$ will not insert into membranes with significantly greater thickness. ${ }^{66-70}$ Fig. 5A shows the current increase after $\alpha$-hemolysin is added to the cis side of the bilayer to a final concentration of $73 \mathrm{nM}$ at approximately $\mathrm{t}=150 \mathrm{~s}$. The concentration is such that it 

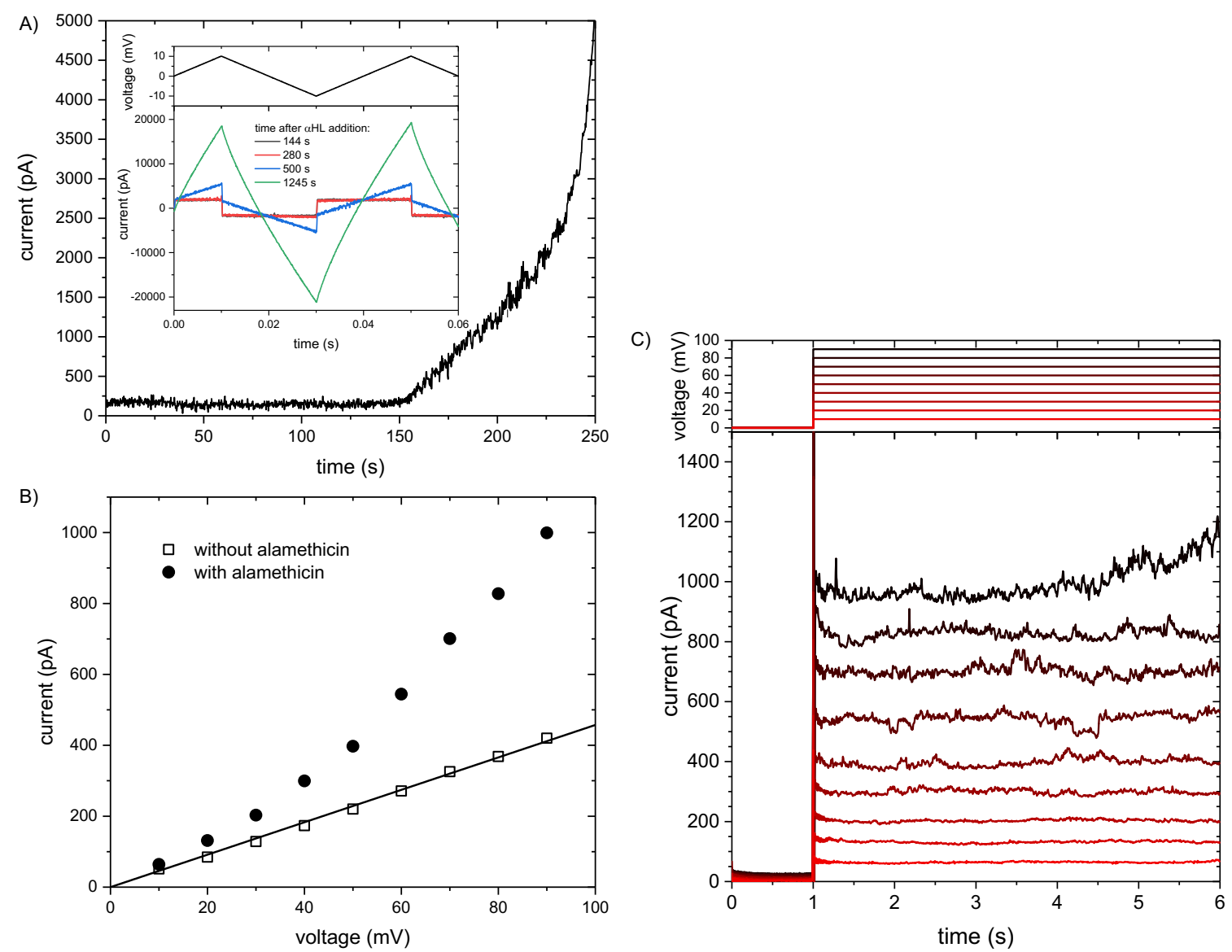

Figure 5: A) Current signal from a DOPC bilayer clamped at $+50 \mathrm{mV}$ with $73 \mathrm{nM} \alpha$ hemolysin added at $t=150 \mathrm{~s}$. Inset: Current response from a $\pm 10 \mathrm{mV}$ triangle wave showing the transition from capacitive response as the $\alpha$-hemolysin $(4.5 \mathrm{nM})$ forms conducting channels. B) Current voltage relationship for a DOPC bilayer with and without alamethicin. C) Current signal from a DOPC bilayer with alamethicin undergoing a step change from 0 to $10-90 \mathrm{mV}$. Each successive line is a $10 \mathrm{mV}$ higher applied field, going from bottom to top.

is difficult to resolve the discrete jumps in current from single pores forming (approximately $5 \mathrm{pA} /$ pore $^{70-72}$, but the expected large increase in current is observed almost immediately. In the inset, a lower concentration of $4.5 \mathrm{nM} \alpha$-hemolysin is added and the response to a \pm $10 \mathrm{mV}$ triangle wave monitored to confirm pore formation. Over time, the current response transitions from purely capacitive to conductive as more pores are inserted into the bilayer.

Alamethicin is a $2 \mathrm{kDa}$ peptide which oligomerizes in lipid membranes. It is voltage gated and exhibits multi-conductance states, which means that upon increasing potential 
the channel open probability increases with a 20 - 400 pA conductance dependent on the number of monomers forming the channel ${ }^{73-76}$. After adding alamethicin to the cis side of the bilayer, the current through the membrane is monitored as the voltage is stepped from $0 \mathrm{mV}$ to increasingly larger potentials. The current-voltage relationship for a DOPC bilayer with and without alamethicin clearly shows the opening of channels with voltage (Fig. 5C). Above $20 \mathrm{mV}$, the current diverges as the voltage causes a higher probability of alamethicin channel opening, which agrees with previous literature results on vesicles ${ }^{77}$ and $\mathrm{BLMs}^{78}$. As Fig. 5C shows, when the applied potential increases from +10 to $+100 \mathrm{mV}$ there are clearly more spikes in current, indicating successful incorporation of alamethicin.

These experiments are an important demonstration of using membrane proteins with the LAMB platform. The use of oil solvent to form the bilayer does not hinder the ability for ion channels to insert into the membrane, and current recordings are able to be made. Although the millimeter scale area of the membrane is an advantage in most cases, an additional challenge to doing single-channel recording experiments in such a setup is to carefully tune the experimental parameters such that only a single channel is detected. Indeed, over time when adding either protein as the concentration increases the current increases above the recording threshold. However, with careful tuning of the stock concentration and washing the membrane with fresh buffer solution, it should be possible to control the concentration of membrane proteins in the bilayer by measuring the discrete increases in current that mark insertion occurrences.

\section{Conclusion}

To better understand biophysical processes, model membrane experiments must faithfully reproduce critical aspects of true cell membranes. Towards this end, we have shown that membranes formed by the LAMB technique have specific capacitance and thicknesses comparable to solvent-free fabrication methods, faithfully contain the expected lipid mole fractions 
based on the bulk compositions, and are able to incorporate model membrane proteins. In particular, the effect of oil not only dictates the static membrane thickness, but also the manner in which the membrane responds to external potentials. This is particularly important when calculating the compressive modulus, which increased an order of magnitude when going from hexadecane to squalene as the oil, and when studying electrostriction due to any cause more generally. While the specific capacitance is easily compared across several methods since it is only based on measured quantities, quantifying the membrane thickness is more difficult since a membrane dielectric constant must be assumed. Using a value of $\varepsilon_{r}$ $=2.5$ is both in line with SAXS data and prior capacitance measurements on bilayers. It is worth noting that since the compressive modulus is based on $\Delta d / d$, the dielectric constant does have a slight effect on the calculated moduli, but it is much less than the effect of oil. Since cell membranes possess a wide diversity of phospholipid species and large concentrations of membrane proteins, it is essential to successfully incorporate these components. Both were demonstrated successfully, showing the potential for the LAMB platform to continue to be applied to intricate problems in the field of membrane biophysics.

\section{Acknowledgement}

We thank Werner Schmidheiny, Christian Furrer, and Kirill Feldman for support in designing and fabricating the experimental sample chamber. This work was supported by ETH Zürich Career Seed Grant SEED-78 16-1, ETH Zürich Grant ETH-03 15-2, and SNSF Grant 200021165974. 


\section{References}

1. Bayley, H. Membrane-protein structure: Piercing insights. Nature 2009, 459, 651-652.

2. Rodriguez-Larrea, D.; Bayley, H. Multistep protein unfolding during nanopore translocation. Nat Nano 2013, 8, 288-295.

3. Bruno, M. J.; Rusinova, R.; Gleason, N. J.; Koeppe II, R. E.; Andersen, O. S. Interactions of drugs and amphiphiles with membranes: modulation of lipid bilayer elastic properties by changes in acyl chain unsaturation and protonation. Faraday Discuss. 2013, 161, 461-480.

4. Lingwood, D.; Simons, K. Lipid Rafts As a Membrane-Organizing Principle. Science 2009, 327, 46-50.

5. Ganesan, P. V.; Boxer, S. G. A membrane interferometer. P. Natl. Acad. Sci. U.S.A. 2009, 106, 5627-5632.

6. Geng, J.; Kim, K.; Zhang, J.; Escalada, A.; Tunuguntla, R.; Comolli, L. R.; Allen, F. I.; Shnyrova, A. V.; Cho, K. R.; Munoz, D. et al. Stochastic transport through carbon nanotubes in lipid bilayers and live cell membranes. Nature 2014, 514, 612-615.

7. Takei, T.; Yaguchi, T.; Fujii, T.; Nomoto, T.; Toyota, T.; Fujinami, M. Measurement of membrane tension of free standing lipid bilayers via laser-induced surface deformation spectroscopy. Soft Matter 2015, 11, 8641-8647.

8. Bayley, H.; Cronin, B.; Heron, A.; Holden, M. A.; Hwang, W. L.; Syeda, R.; Thompson, J.; Wallace, M. Droplet interface bilayers. Mol. BioSyst. 2008, 4, 1191-1208.

9. Fischer, A.; Holden, M. A.; Pentelute, B. L.; Collier, R. J. Ultrasensitive detection of protein translocated through toxin pores in droplet-interface bilayers. P. Natl. Acad. Sci. U.S.A. 2011, 108, 16577-16581. 
10. Leptihn, S.; Castell, O. K.; Cronin, B.; Lee, E.-H.; Gross, L. C. M.; Marshall, D. P.; Thompson, J. R.; Holden, M.; Wallace, M. I. Constructing droplet interface bilayers from the contact of aqueous droplets in oil. Nature Protocols 2013, 8, 1048-1057.

11. Thompson, J. R.; Heron, A. J.; Santoso, Y.; Wallace, M. I. Enhanced Stability and Fluidity in Droplet on Hydrogel Bilayers for Measuring Membrane Protein Diffusion. Nano Lett 2007, 7, 3875-3878.

12. Gross, L. C. M.; Heron, A. J.; Baca, S. C.; Wallace, M. I. Determining Membrane Capacitance by Dynamic Control of Droplet Interface Bilayer Area. Langmuir 2011, 27, $14335-14342$.

13. Taylor, G. J.; Venkatesan, G. A.; Collier, C. P.; Sarles, S. A. Direct in situ measurement of specific capacitance, monolayer tension, and bilayer tension in a droplet interface bilayer. Soft Matter 2015, 11, 7592-7605.

14. White, S. H. Phase transitions in planar bilayer membranes. Biophys. J. 1975, 15, 95117.

15. Schuster, B.; Pum, D.; Braha, O.; Bayley, H.; Sleytr, U. B. Self-assembled $\alpha$-hemolysin pores in an S-layer-supported lipid bilayer. Biochimica et Biophysica Acta (BBA) Biomembranes 1998, 1370, 280-288.

16. White, S. H. Temperature-dependent structural changes in planar bilayer membranes: Solvent "freeze-out". Biochimica et Biophysica Acta (BBA) - Biomembranes 1974, 356, $8-16$.

17. Batishchev, O. V.; Indenbom, A. V. Alkylated glass partition allows formation of solventfree lipid bilayer by Montal-Mueller technique. Bioelectrochemistry 2008, 74, 22-25.

18. White, S. H. Formation of "solvent-free" black lipid bilayer membranes from glyceryl monooleate dispersed in squalene. Biophys. J. 1978, 23, 337-347. 
19. Castellana, E. T.; Cremer, P. S. Solid supported lipid bilayers: From biophysical studies to sensor design. Surface Science Reports 2006, 61, 429-444.

20. Zhang, L.; Granick, S. Slaved diffusion in phospholipid bilayers. P. Natl. Acad. Sci. U.S.A. 2005, 102, 9118-9121.

21. Kukura, P.; Ewers, H.; Müller, C.; Renn, A.; Helenius, A.; Sandoghdar, V. High-speed nanoscopic tracking of the position and orientation of a single virus. Nature Methods 2009, 6, 923-927.

22. Hemmerle, A.; Malaquin, L.; Charitat, T.; Lecuyer, S.; Fragneto, G.; Daillant, J. Controlling interactions in supported bilayers from weak electrostatic repulsion to high osmotic pressure. P. Natl. Acad. Sci. U.S.A. 2012, 109, 19938-19942.

23. Pace, H.; Simonsson Nyström, L.; Gunnarsson, A.; Eck, E.; Monson, C.; Geschwindner, S.; Snijder, A.; Höök, F. Preserved Transmembrane Protein Mobility in PolymerSupported Lipid Bilayers Derived from Cell Membranes. Anal Chem 2015, 87, 91949203.

24. Richards, M. J.; Hsia, C.-Y.; Singh, R. R.; Haider, H.; Kumpf, J.; Kawate, T.; Daniel, S. Membrane Protein Mobility and Orientation Preserved in Supported Bilayers Created Directly from Cell Plasma Membrane Blebs. Langmuir 2016, 32, 2963-2974.

25. Evans, E.; Rawicz, W. Entropy-driven tension and bending elasticity in condensed-fluid membranes. Phys. Rev. Lett. 1990, 64, 2094-2097.

26. Baumgart, T.; Hess, S. T.; Webb, W. W. Imaging coexisting fluid domains in biomembrane models coupling curvature and line tension. Nature 2003, 425, 821-824.

27. Veatch, S. L.; Keller, S. L. Separation of Liquid Phases in Giant Vesicles of Ternary Mixtures of Phospholipids and Cholesterol. Biophys. J. 2003, 85, 3074-3083. 
28. Dimova, R.; Bezlyepkina, N.; Jordö, M. D.; Knorr, R. L.; Riske, K. A.; Staykova, M.; Vlahovska, P. M.; Yamamoto, T.; Yang, P.; Lipowsky, R. Vesicles in electric fields: Some novel aspects of membrane behavior. Soft Matter 2009, 5, 3201-3212.

29. Dezi, M.; Di Cicco, A.; Bassereau, P.; Lévy, D. Detergent-mediated incorporation of transmembrane proteins in giant unilamellar vesicles with controlled physiological contents. P. Natl. Acad. Sci. U.S.A. 2013, 110, 7276-7281.

30. Dong Chen, M. M. S. Large effect of membrane tension on the fluid-solid phase transitions of two-component phosphatidylcholine vesicles. P. Natl. Acad. Sci. U.S.A. 2014, $111,179-184$.

31. Evans, E.; Rawicz, W.; Smith, B. A. Concluding remarks Back to the future: mechanics and thermodynamics of lipid biomembranes. Faraday Discuss. 2013, 161, 591-611.

32. Mosgaard, L. D.; Zecchi, K. A.; Heimburg, T. Mechano-capacitive properties of polarized membranes. Soft Matter 2015, 11, 7899-7910.

33. Kamiya, K.; Kawano, R.; Osaki, T.; Akiyoshi, K.; Takeuchi, S. Cell-sized asymmetric lipid vesicles facilitate the investigation of asymmetric membranes. Nature Chem $\mathbf{2 0 1 6}$, 8, 881-889.

34. Heberle, F. A.; Marquardt, D.; Doktorova, M.; Geier, B.; Standaert, R. F.; Heftberger, P.; Kollmitzer, B.; Nickels, J. D.; Dick, R. A.; Feigenson, G. W. et al. Subnanometer Structure of an Asymmetric Model Membrane: Interleaflet Coupling Influences Domain Properties. Langmuir 2016, 32, 5195-5200.

35. Karamdad, K.; Law, R. V.; Seddon, J. M.; Brooks, N. J.; Ces, O. Studying the effects of asymmetry on the bending rigidity of lipid membranes formed by microfluidics. Chem Commun 2016, 52, 5277-5280. 
36. Najem, J. S.; Dunlap, M. D.; Rowe, I. D.; Freeman, E. C.; Grant, J. W.; Sukharev, S.; Leo, D. J. Activation of bacterial channel MscL in mechanically stimulated droplet interface bilayers. Scientific Reports 2015, 5, 13726.

37. Sparr, E.; Hallin, L.; Markova, N.; Wennerström, H. Phospholipid-Cholesterol Bilayers under Osmotic Stress. Biophys. J. 2002, 83, 2015-2025.

38. Beltramo, P. J.; Van Hooghten, R.; Vermant, J. Millimeter-area, free standing, phospholipid bilayers. Soft Matter 2016, 12, 4324-4331.

39. Beltramo, P. J.; Vermant, J. Simple Optical Imaging of Nanoscale Features in FreeStanding Films. ACS Omega 2016, 1, 363-370.

40. Pereira, L.; Johansson, C.; Blanch, H. W.; Radke, C. J. A bike-wheel microcell for measurement of thin-film forces. Colloids Surf., A 2001, 186, 103-111.

41. Gramse, G.; Dols-Perez, A.; Edwards, M. A.; Fumagalli, L.; Gomila, G. Nanoscale Measurement of the Dielectric Constant of Supported Lipid Bilayers in Aqueous Solutions with Electrostatic Force Microscopy. Biophys. J. 2013, 104, 1257-1262.

42. Hanai, T.; Haydon, D. A.; Taylor, J. Polar group orientation and the electrical properties of lecithin bimolecular leaflets. Journal of Theoretical Biology 1965, 9, 278-296.

43. White, S. H. The surface charge and double layers of thin lipid films formed from neutral lipids. Biochimica et Biophysica Acta (BBA) - Biomembranes 1973, 323, 343-350.

44. Bergeron, V.; Radke, C. J. Equilibrium Measurements of Oscillatory Disjoining Pressures in Aqueous Foam Films. Langmuir 1992, 8, 3020-3026.

45. Claesson, P. M.; Ederth, T.; Bergeron, V.; Rutland, M. W. Techniques for measuring surface forces. Adv Colloid Interfac 1996, 67, 119-183.

46. Zhang, Y.; Sharma, V. Domain expansion dynamics in stratifying foam films: experiments. Soft Matter 2015, 11, 4408-4417. 
47. Montal, M.; Mueller, P. Formation of Bimolecular Membranes From Lipid Monolayers and a Study of Their Electrical Properties. P. Natl. Acad. Sci. U.S.A. 1972, 69, 35613566.

48. Valincius, G.; Heinrich, F.; Budvytyte, R.; Vanderah, D. J.; McGillivray, D. J.; Sokolov, Y.; Hall, J. E.; L sche, M. Soluble Amyloid ?-Oligomers Affect Dielectric Membrane Properties by Bilayer Insertion and Domain Formation: Implications for Cell Toxicity. Biophys. J. 2008, 95, 4845-4861.

49. Lewis, B. A.; Engelman, D. M. Lipid bilayer thickness varies linearly with acyl chain length in fluid phosphatidylcholine vesicles. Journal of Molecular Biology 1983, 166, $211-217$.

50. Balgavý, P.; Dubničková, M.; Kučerka, N.; Kiselev, M. A.; Yaradaikin, S. P.; Uhrikova, D. Bilayer thickness and lipid interface area in unilamellar extruded 1, 2diacylphosphatidylcholine liposomes: a small-angle neutron scattering study. Biochimica et Biophysica Acta (BBA) - Bioenergetics 2001, 1512, 40-52.

51. Kučerka, N.; Tristram-Nagle, S.; Nagle, J. F. Structure of Fully Hydrated Fluid Phase Lipid Bilayers with Monounsaturated Chains. J Membrane Biol 2006, 208, 193-202.

52. Pan, J.; Tristram-Nagle, S.; Kučerka, N.; Nagle, J. F. Temperature Dependence of Structure, Bending Rigidity, and Bilayer Interactions of Dioleoylphosphatidylcholine Bilayers. Biophys. J. 2008, 94, 117-124.

53. Kučerka, N.; Nieh, M.-P.; Katsaras, J. Fluid phase lipid areas and bilayer thicknesses of commonly used phosphatidylcholines as a function of temperature. Biochimica et Biophysica Acta (BBA) - Biomembranes 2011, 1808, 2761-2771.

54. White, S. H. Analysis of the Torus Surrounding Planar Lipid Bilayer Membranes. Biophys. J. 1972, 12, 432-445. 
55. Heimburg, T. The Capacitance and Electromechanical Coupling of Lipid Membranes Close to Transitions: The Effect of Electrostriction. Biophys. J. 2012, 103, 918-929.

56. Hianik, T.; Dlugopolsky, J.; Gyepessova, M. Electrostriction of lipid bilayers on a solid support. Influence of hydrocarbon solvent and d.c. voltage. Bioelectrochemistry and Bioenergetics 1993, 31, 99-111.

57. Hianik, T.; Dlugopolsky, J.; Passechnik, V. I.; Sargent, D. F.; Ivanov, S. A. Electrostriction and membrane potential of lipid bilayers on a metal support. Colloids Surf., A 1996, 106, 109-118.

58. Garten, M.; Mosgaard, L. D.; Bornschlögl, T.; Dieudonné, S.; Bassereau, P.; Toombes, G. E. S. Whole-GUV patch-clamping. P. Natl. Acad. Sci. U.S.A. 2017, 114, 328-333.

59. Ziebert, F.; Lacoste, D. A Poisson-Boltzmann approach for a lipid membrane in an electric field. New J. Phys. 2010, 12, 095002.

60. Hianik, T. Electrostriction and dynamics of solid supported lipid films. Reviews in Molecular Biotechnology 2000, 74, 189-205.

61. Krupa, I.; Luyt, A. S. Mechanical properties of uncrosslinked and crosslinked linear low-density polyethylene/wax blends. J. Appl. Polym. Sci. 2001, 81, 973-980.

62. Kučerka, N.; Liu, Y.; Chu, N.; Petrache, H. I.; Tristram-Nagle, S.; Nagle, J. F. Structure of Fully Hydrated Fluid Phase DMPC and DLPC Lipid Bilayers Using X-Ray Scattering from Oriented Multilamellar Arrays and from Unilamellar Vesicles. Biophys. J. 2005, 88, 2626-2637.

63. Pan, J.; Heberle, F. A.; Tristram-Nagle, S.; Szymanski, M.; Koepfinger, M.; Katsaras, J.; Kučerka, N. Molecular structures of fluid phase phosphatidylglycerol bilayers as determined by small angle neutron and X-ray scattering. Biochimica et biophysica acta $\mathbf{2 0 1 2}$, $1818,2135-2148$. 
64. Pan, J.; Marquardt, D.; Heberle, F. A.; Kučerka, N.; Katsaras, J. Revisiting the bilayer structures of fluid phase phosphatidylglycerol lipids: Accounting for exchangeable hydrogens. Biochimica et Biophysica Acta (BBA) - Biomembranes 2014, 1838, 2966-2969.

65. Song, L.; Hobaugh, M. R.; Shustak, C.; Cheley, S.; Bayley, H.; Gouaux, J. E. Structure of Staphylococcal $\alpha$-Hemolysin, a Heptameric Transmembrane Pore. Science 1996, 274, $1859-1865$.

66. Malmstadt, N.; Nash, M. A.; Purnell, R. F.; Schmidt, J. J. Automated Formation of Lipid-Bilayer Membranes in a Microfluidic Device. Nano Lett 2006, 6, 1961-1965.

67. Funakoshi, K.; Suzuki, H.; Takeuchi, S. Lipid Bilayer Formation by Contacting Monolayers in a Microfluidic Device for Membrane Protein Analysis. Anal Chem 2006, 78, 8169-8174.

68. Gornall, J. L.; Mahendran, K. R.; Pambos, O. J.; Steinbock, L. J.; Otto, O.; Chimerel, C.; Winterhalter, M.; Keyser, U. F. Simple Reconstitution of Protein Pores in Nano Lipid Bilayers. Nano Lett 2011, 11, 3334-3340.

69. Czekalska, M. A.; Kaminski, T. S.; Jakiela, S.; Sapra, K. T.; Bayley, H.; Garstecki, P. A droplet microfluidic system for sequential generation of lipid bilayers and transmembrane electrical recordings. Lab Chip 2014, 15, 541-548.

70. Marin, V.; Kieffer, R.; Padmos, R.; Aubin-Tam, M.-E. Stable Free-Standing Lipid Bilayer Membranes in Norland Optical Adhesive 81 Microchannels. Anal Chem 2016, 88, 74667470.

71. Suzuki, H.; Tabata, K. V.; Noji, H.; Takeuchi, S. Electrophysiological recordings of single ion channels in planar lipid bilayers using a polymethyl methacrylate microfluidic chip. Biosensors and Bioelectronics 2007, 22, 1111-1115. 
72. Osaki, T.; Takeuchi, S. Artificial Cell Membrane Systems for Biosensing Applications. Anal Chem 2017, 89, 216-231.

73. Woolley, G. A.; Wallace, B. A. Model ion channels: Gramicidin and alamethicin. $J$ Membrane Biol 1992, 129, 109-136.

74. Sansom, M. S. P. Alamethicin and related peptaibols - model ion channels. Eur Biophys $J$ 1993, 22, 105-124.

75. Hamill, O. P.; Martinac, B. Molecular basis of mechanotransduction in living cells. Physiological reviews 2001,

76. Mayer, M.; Kriebel, J. K.; Tosteson, M. T.; Whitesides, G. M. Microfabricated Teflon Membranes for Low-Noise Recordings of Ion Channels in Planar Lipid Bilayers. Biophys. J. 2003, 85, 2684-2695.

77. Archer, S. J.; Cafiso, D. S. Voltage-dependent conductance for alamethicin in phospholipid vesicles. A test for the mechanism of gating. Biophys. J. 1991, 60, 380-388.

78. Cafiso, D. S. Alamethicin: A Peptide Model for Voltage Gating and Protein-Membrane Interactions. Annu. Rev. Biophys. Biomol. Struct. 1994, 23, 141-165. 


\section{For Table of Contents Only}

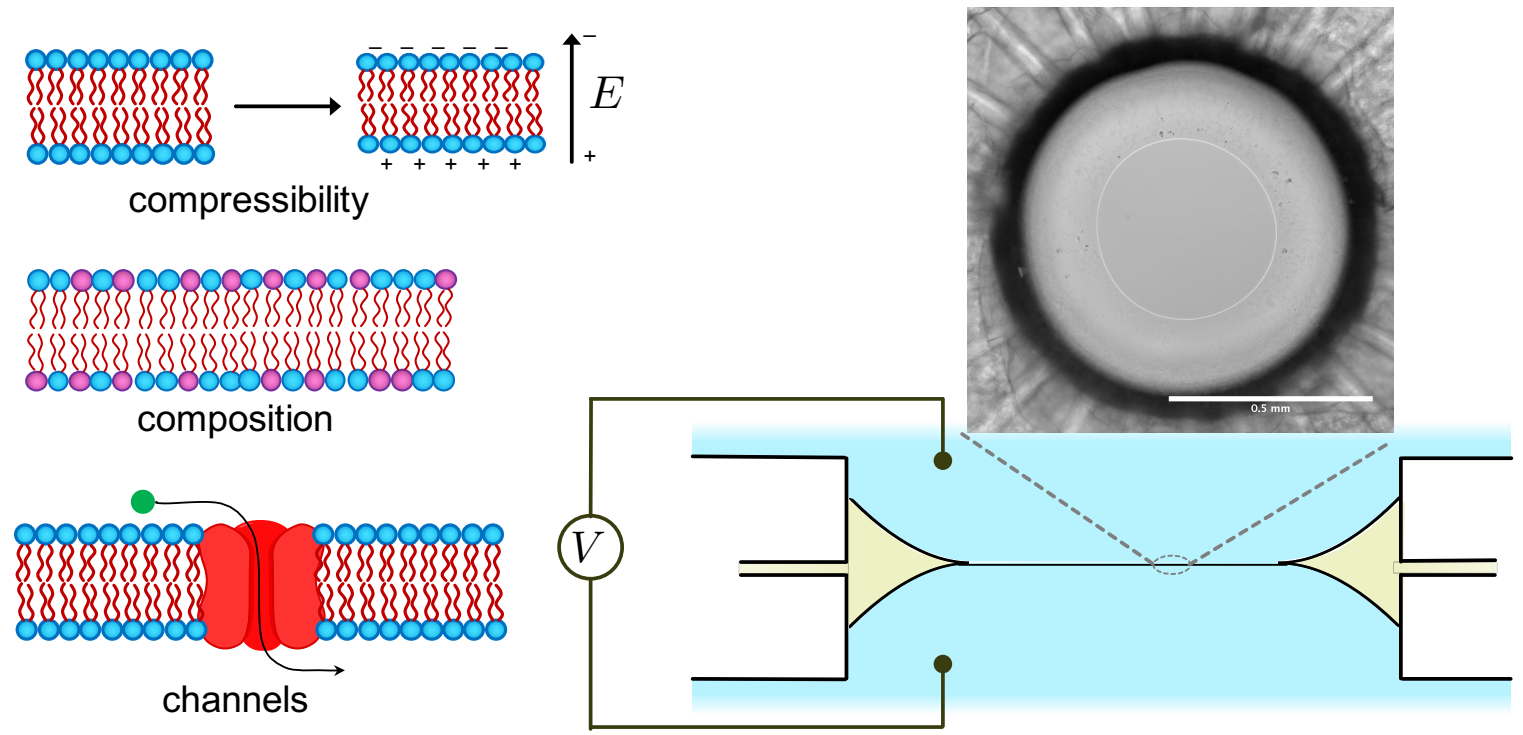

